\title{
AUTOPERCEPÇÃO DOS ESTUDANTES SOBRE A PARTICIPAÇÃO EM ATIVIDADES EXTRACURRICULARES DE TRANSFERÊNCIA DE CONHECIMENTO: O EXEMPLO DA TRANSIÇÃO SEGURA
}

\author{
Cristina Lavareda Baixinho ${ }^{1,2}$, Óscar Ferreira', Mário Cardoso ${ }^{1}$, Patricia Nascimento ${ }^{3}$, Rita \\ Pedrosa $^{3}$, Paulo Gonçalves ${ }^{3}$ \\ ${ }^{1}$ Escola Superior de Enfermagem de Lisboa; crbaixinho@esel.pt; oferreira@esel.pt; mmcardoso@esel.pt \\ 2 ciTechCare, Leiria, Portugal \\ ${ }^{3}$ Hospital Vila Franca de Xira; patricia.nascimento@hvfx.pt; ritacardosopedrosa@gmail.com; paulo5cpleer@gmail.com
}

\begin{abstract}
Resumo. Introdução: A emergência de um novo paradigma na formação na área da saúde apela ao aumento das competências associadas à ciência, para que os futuros profissionais tenham conhecimento sobre métodos e técnicas de investigação e utilizem a literacia científica como uma ferramenta de trabalho. Objetivos: Compreender como os estudantes percepcionam o seu envolvimento nas atividades do projeto da Transição Segura e o contributo para o desenvolvimento de uma prática baseada na evidência. Métodos: Focus Group com oito estudantes finalistas de enfermagem, que respeitavam os critérios de inclusão e que participaram no projeto. A análise qualitativa dos achados seguiu as etapas da codificação; armazenamento/recuperação e interpretação. Estudo aprovado por uma Comissão de Ética. Resultados: A análise do discurso dos participantes permitiu a definição de cinco categorias sobre o seu envolvimento no projeto: trabalho interdisciplinar, integração teórico-prática, tomada de decisão clínica baseada na evidência, autoaprendizagem e literacia científica e respectivas subcategorias. Conclusões: $\mathrm{O}$ envolvimento dos estudantes em projetos de translação do conhecimento para a clínica contribui para a sua aprendizagem, promove a tomada de decisão baseada na evidência, desenvolve competências de pesquisa, análise e transferência do conhecimento para a práxis.
\end{abstract}

Palavras-chave: Estudantes; Enfermagem; Prática Clínica Baseada em Evidências; Aprendizagem; Gestão do Conhecimento.

\section{STUDENTS' SELF-PERCEPTION ABOUT PARTICIPATING IN EXTRACURRICULAR KNOWLEDGE TRANSFER ACTIVITIES: THE EXAMPLE OF THE SAFE TRANSITION}

\begin{abstract}
Introduction: The emergence of a new paradigm in health training calls for an increase in the skills associated with science, so that future professionals have knowledge about research methods and techniques and use scientific literacy as a working tool. Goals: Understand how students perceive their involvement in the activities of the Safe Transition project and the contribution to the development of an evidence-based practice. Methods: Focus Group with eight finalist nursing students, who respected the inclusion criteria and who participated in the project. The qualitative analysis of the findings followed the steps of codification; storage / retrieval and interpretation. Study approved by an Ethics Committee. Results: The analysis of the participants discourse allowed the definition of five categories about their involvement in the project: interdisciplinary work, theoretical-practical integration, evidence-based clinical decision making, self-learning and scientific literacy and respective subcategories. Conclusions. Students involvement in knowledge translation projects for the clinic contributes to their learning, promotes evidence-based decision making, develops research, analysis and knowledge transfer skills for praxis.
\end{abstract}

Keywords: Students; Nursing; Evidence-Based Practice; Learning; Knowledge Management. 


\section{INTRODUÇÃO}

O projeto transição segura (TS) é um projeto de Translação do Conhecimento (TC) que envolve, num trabalho em parceria, o Hospital de Vila Franca de Xira (HVFX), a Escola Superior de Enfermagem de Lisboa (ESEL), e o Agrupamento de Centros de Saúde do Estuário do Tejo (ACESET), assenta num processo simultâneo de resolução de problemas, formação, investigação e ação, em que a finalidade primordial é a Translação do Conhecimento para a resolução de problemas dos diferentes serviços de internamento hospitalar para aumentarem o conhecimento e capacitarem os doentes e as suas famílias no processo de transição do hospital para a comunidade, favorecendo circuitos de comunicação que promovam a continuidade de cuidados, diminuindo os reinternamentos após a alta hospitalar, e promovendo a reabilitação e a inserção na comunidade (Paniagua et al., 2018; Ferreira et al., 2019).

A equipa do projeto, desde a sua concepção, tinha a clara consciência das dificuldades existentes para a integração teórico-prática e transferência do conhecimento para a práxis. O envolvimento de profissionais das áreas clínicas, académicos e estudantes de enfermagem, teve o intuito de promover uma Prática Baseada na Evidência (PBE), desde a produção à utilização do conhecimento emergente nas áreas clínicas (Baixinho, Ferreira, Marques, Presado, \& Cardoso, 2017; Baixinho \& Ferreira, 2019).

Esta preocupação não é exclusiva do grupo de trabalho, como observam Kitson et al. (2018), têm sido criadas muitas representações do movimento do conhecimento em saúde, e múltiplos modelos para transformar a evidência em políticas e práticas, com o propósito de implementar melhorias sustentáveis. Todavia o processo de TC, desde a sua produção à utilização segura, ocorre dentro de um sistema social complexo, onde todo o processo precisa de ser tido em conta, o que justifica per si, a adaptação das atividades de pesquisa às necessidades dos clientes (Baumbush et al., 2008).

No decurso deste projeto, os estudantes, têm tido a possibilidade de o integrar nos serviços de internamento hospitalar (urgência, unidade de cuidados intensivos, medicina, cirurgia e ortopedia) onde decorre o ensino clínico, com a definição de um conjunto de atividades, que são creditadas como um suplemento ao diploma destes futuros profissionais. A opção pelo envolvimento dos estudantes deve-se à concordância que para atingir um nível elevado de aprendizagem, há que investir em ambientes de prática clínica de qualidade e melhorar a supervisão e a avaliação da prática do estudante (Heidari \& Norouzadeh, 2015; Baixinho \& 
Ferreira, 2019), até porque os contextos clínicos, bem como as situações clínicas dos doentes estão a tornar-se mais complexos, exigindo um aumento de competências científicas (Einarsen \& Giske, 2019).

A revisão da literatura permitiu verificar que nos últimos anos têm emergido nos currículos em enfermagem a introdução de educação em PBE, para o desenvolvimento académico dos estudantes e aprofundamento dos conteúdos teóricos (Mena-Tudela et al., 2018).

Apesar das vantagens da formação corroboramos a opinião de Slattery et al., (2016) e de Mena-Tudela et al., (2018) de que a aprendizagem de uma PBE não é feita nos bancos da escola, implica um contacto com projetos de investigação, conhecimento de métodos e técnicas de investigação e capacidade de análise sobre o desenho do estudo, a sua qualidade e os resultados obtidos, ou seja, que o estudante seja um participante ativo em alguma fase do processo de pesquisa. Sun, Liu, Wu e Li, (2014) vão um pouco mais longe ao advogarem que é difícil aprender evidência de um modo tradicional em que um professor prepara aulas, explica e demonstra aos seus alunos, confiando que desta maneira este fique apto para utilizar a investigação e para produzir resultados seguros na clínica.

É consensual que a educação em enfermagem deve fortalecer a capacidade de pensamento crítico e aprimorar as habilidades dos estudantes nas áreas de pensamento analítico, resolução de problemas e raciocínio clínico (Einarsen \& Giske, 2019). Alguns autores reforçam que os Ensinos Clínicos (EC) são momentos-chave para a melhoria dos conhecimentos, atitudes e competências dos alunos ao nível da evidência científica (MenaTudela et al., 2018; Kitson et al. (2018), considerando que a aprendizagem sobre investigação e utilização do conhecimento científico é maior quando é integrada nesta tipologia de ensino. Slattery et al. (2016) consideram que para além de aprender teoria sobre investigação e familiarizar-se com trabalho interprofissional a este nível, importa que os estudantes participem em pesquisa, interajam efetivamente com investigadores académicos e clínicos e incorporarem os resultados da investigação nas intervenções de enfermagem e isso é possível durante as experiências que são proporcionadas aos estudantes no contexto de EC.

Face ao exposto, é objetivo deste estudo compreender como os estudantes percecionam o seu envolvimento nas atividades do projeto da Transição Segura e o contributo para o desenvolvimento de uma prática baseada na evidência. 


\section{METODOLOGIA}

Estudo qualitativo, transversal, descritivo e exploratório que recorreu ao focus-group como método para responder à questão de pesquisa. A opção por uma abordagem qualitativa deve-se à natureza e o estado da arte sobre o tema. A revisão da literatura permitiu aferir que há poucos estudos sobre a participação dos estudantes, da formação pré-graduada em enfermagem, em projetos de investigação. Por outro lado, a singularidade da experiência que foi proporcionada justificava que se explorasse a autoperceção das vantagens, ou não, da mesma e o seu contributo para a aprendizagem da PBE.

Silva, Veloso e Keating (2014) observam que este é um método de investigação dirigido à recolha de dados, que possibilita localizar a interação na discussão do grupo como a fonte dos dados e, reconhece o papel ativo do investigador na dinamização da discussão do grupo para efeitos de recolha dos dados.

A partir da questão "Quais as vantagens da participação dos estudantes de enfermagem no projeto da Transição Segura para o desenvolvimento de conhecimentos, atitudes e competências de utilização da evidência?", definiu-se o desenho do estudo e subsequentemente o protocolo para o focus group que foi organizado em cinco fases: 1) planeamento, 2) preparação, 3) moderação, 4) análise dos dados e 5) divulgação dos resultados (Silva, Veloso, \& Keating, 2014). Após a definição da problemática e da revisão da literatura aferiu-se o objetivo do focus group em consonância com as finalidades do estudo e elaborou-se um guião para a entrevista semiestruturada. Optou-se por estabelecer cinco questões gerais sobre as possibilidades que o envolvimento no projeto trouxe, ou não, em termos de trabalho interdisciplinar, pesquisa de evidência, utilização dos resultados de investigação nas suas intervenções, desenvolvimento de competências que envolvam investigação e transferência da evidência para os contextos de clínica.

Estas perguntas serviram como um guião para orientar a entrevista, prevendo-se atempadamente que à medida que a discussão avançasse, iriam surgir questões mais específicas em função dos contributos do grupo. Como referem Krueger e Casey (2014) é possível 'afunilar' as questões iniciais, mais genéricas, que têm um papel primordial para ajudarem os participantes a falarem e a pensarem sobre o tópico. Tal abordagem, de compromisso, torna assim possível aceder, por um lado, às perspectivas dos próprios participantes na primeira parte de cada discussão e, por outro, às respostas a interesses específicos do investigador na segunda parte (Silva, Veloso, \& Keating, 2014). Os 
participantes foram selecionados de entre os estudantes envolvidos no projeto TS, no segundo semestre, do ano letivo 2018/2019. Tal como em outros métodos qualitativos, os focus group assentam em amostras intencionais, selecionando os participantes que à partida se prevê que gerem as discussões mais produtivas (Silva, Veloso \& Keating, 2014). Foram critérios de inclusão terem realizado o último EC da sua licenciatura numa das instituições parceiras do projeto, estarem envolvidos num dos projetos em curso nos diferentes serviços, não terem reprovado a esta unidade curricular em anos anteriores e manifestarem interesse em participar. Esta foi uma amostra homogénea que possibilitou a focalização na discussão do fenómeno em estudo, já que os participantes têm em comum uma característica relevante face ao tema em discussão - o terem participado num projeto de translação do conhecimento para a clínica, o que reforça as observações de Krueger e Casey (2009) sobre a seleção dos constituintes do grupo de discussão.

O número de participantes foi definido a priori tendo em conta as recomendações dos autores que defendem que o número ideal de participantes se situa entre cinco a dez participantes (Krueger \& Casey, 2014), optando-se por 8 estudantes. No inicio do EC todos os estudantes envolvidos no projeto estavam informados que no final poderiam participar de forma livre esclarecida num focus-group. Duas semanas antes do final do EC foi enviada informação com a data do mesmo e confirmado se mantinham o interesse em participar. Dos 15 estudantes em EC 14 manifestaram interesse. Desses selecionou-se aleatoriamente o grupo final.

Uma vez que esta era uma atividade extracurricular que decorreu em simultâneo com um EC do qual resultava uma nota final, o focus group só foi realizado após a saída da nota da unidade curricular para minimizar o efeito de respostas 'socialmente desejáveis'. A recolha de achados foi gravada (vídeo), decorreu na escola, numa sala que foi organizada para o efeito, com o intuito de criar um ambiente confortável para facilitar a participação, evitar interrupções e garantir o sigilo da informação (Krueger \& Casey, 2014; Silva, Veloso, \& Keating, 2014).

No e-mail enviado e no inicio do focus group os participantes foram informados sobre os objetivos e as regras de participação, incluindo tempo estimado de duração (75 minutos) de modo a evitar abandonos precoces aquando a discussão em grupo (Silva, Veloso \& Keating, 2014), foi identificado o nome do moderador e do co-moderador da sessão. 
O papel do moderador constitui um elemento chave nesta fase (Kueger \& Casey, 2014) não só para o suporte ao grupo na exploração do tópico, mas também pela possibilidade de poderem emergir novos insights face ao tema (Silva, Veloso, \& Keating, 2014). As questões norteadoras e o trabalho do moderador foi revisto previamente pela equipa para garantir as competências de moderação, de dinâmica de grupo, de controlo de possíveis elementos críticos para garantir o sucesso (Silva, Veloso, \& Keating, 2014). Como sublinham Krueger e Casey (2014) o papel do moderador não é o de emitir julgamentos, mas o de questionar, ouvir, manter a discussão alinhada com os objetivos/questões e certificar-se que cada participante tem oportunidade de participar.

A opção por um co-moderador foi consonante com as indicações de Krueger e Casey (2014) para aumentar o rigor no processo em que o moderador, tem como principal missão a condução e a manutenção da discussão e o segundo elemento auxilia a moderação, faz a gestão do equipamento de gravação, está atento às condições logísticas e do ambiente físico, dá resposta a interrupções inesperadas e toma notas sobre a discussão do grupo (Krueger \& Casey, 2014). No nosso caso, o segundo elemento fez ainda um registo da sequência das intervenções, das dúvidas, de informações privilegiadas sobre expressões faciais, gestos, tom de voz, os momentos em que os discursos foram interrompidos pelos outros participantes e os sentimentos que apareceram ao longo da atividade.

A gravação foi ouvida duas vezes antes da transcrição. A transcrição foi efetuada por um dos investigadores presentes de forma a permitir "visualizar" o que ocorreu no grupo; uma das tarefas mais intensas em termos de tempo deste método de recolha de informação prende-se exatamente com o processo de transcrição (Silva, Veloso, \& Keating, 2014).

A análise qualitativa dos achados seguiu as etapas definidas por Bloor, Frankland, Thomas e Robson (2001): 1) codificação para a atribuição de categorias, refletindo estas os temas presentes no guião, bem como os novos, que emergiram da discussão dos grupos; 2) armazenamento/recuperação: esta fase é dedicada à compilação de todos os extractos do texto subordinados à mesma categoria de modo a poder compará-los; 3) interpretação: a partir da análise sistemática dos dados. Na definição das categorias foi assegurada a representatividade, a exaustividade, a homogeneidade e a pertinência.

A análise dos achados foi feita com recurso a software informático (NVivo) para facilitar o processo e aumentar o rigor. 
Este estudo está autorizado por uma Comissão de Ética. As instituições envolvidas têm um protocolo de parceria formalizado que autoriza a realização de investigação. Foram respeitados todos os pressupostos éticos e formais desde a autorização dos estudantes para integrarem o projeto, enquanto atividade extracurricular, às questões éticas inerentes ao desenvolvimento da investigação. Foi assegurado a todos os participantes o anonimato e a confidencialidade dos dados.

\section{RESULTADOS}

Participaram neste estudo 8 estudantes finalistas do curso de licenciatura em enfermagem, maioritariamente mulheres (06), com uma média de idades de 22,3 anos.

A análise do discurso dos participantes neste focus group permitiu a definição de cinco categorias sobre o envolvimento dos estudantes no projeto TS: trabalho interdisciplinar, integração teórico-prática, tomada de decisão clínica baseada na evidência, autoaprendizagem e literacia científica e respectivas subcategorias (Tabela 1).

Tabela 1. Corpus da análise de conteúdo. Lisboa; Portugal. 2020.

\begin{tabular}{|c|c|c|}
\hline Categoria & Subcategoria & Unidades de Registo \\
\hline \multirow[t]{4}{*}{ Trabalho interdisciplinar } & Integração & 11 \\
\hline & Trabalho em equipa & 9 \\
\hline & Interdisciplinaridade & 6 \\
\hline & Subtotal & 26 \\
\hline \multirow[t]{4}{*}{ Integração teórico-prática } & Integração dos conteúdos teóricos & 13 \\
\hline & Conhecimento na ação & 5 \\
\hline & Adequação ao contexto & 4 \\
\hline & Subtotal & 22 \\
\hline \multirow{6}{*}{$\begin{array}{l}\text { Tomada de decisão clínica } \\
\text { baseada na evidência }\end{array}$} & Intencionalidade na execução & 9 \\
\hline & Antecipação do resultado & 8 \\
\hline & Reflexão sobre a ação & 7 \\
\hline & Capacidade de resolução de problemas & 7 \\
\hline & Negociação da decisão em equipa & 7 \\
\hline & Subtotal & 38 \\
\hline \multirow[t]{4}{*}{ Autoaprendizagem } & Autonomia e responsabilidade & 8 \\
\hline & Motivação para aprender & 4 \\
\hline & Saber questionar & 3 \\
\hline & Subtotal & 15 \\
\hline \multirow[t]{6}{*}{ Literacia científica } & Transferir conhecimento & 10 \\
\hline & Avaliar investigação & 8 \\
\hline & Interpretar resultados & 6 \\
\hline & Comunicar ciência & 4 \\
\hline & Subtotal & 33 \\
\hline & Total & 134 \\
\hline
\end{tabular}


O Trabalho interdisciplinar emerge como uma vantagem clara da participação no projeto TS. Os participantes consideram que o fato de, em alguns serviços, as equipas multidisciplinares estarem envolvidas no projeto promove uma integração rápida e com maior compromisso dos profissionais em suportar não só a atividade extracurricular, mas também o concretizar dos resultados de aprendizagem previstos para o EC. Por outro lado, desde os primeiros dias de EC há atividades da TS, o que leva a que "desde o inicio fui aceite como uma deles, não era a estudante, era um elemento da equipa com funções e trabalho igual... não havia diferenciação" (E4), o que contribuiu para diminuir a "incerteza acrescida que acompanha todos os períodos de EC, pelo desconhecimento das equipas, das suas dinâmicas e das expectativas que tem para o nosso desempenho"(E7).

O percurso iniciado com a integração nos serviços possibilita o trabalho interdisciplinar com os profissionais para um fim comum e transversal ao conteúdo funcional das diferentes categorias profissionais "fiquei surpresa quando me disseram para sentar e começaram a falar comigo de igual para igual, queriam saber a minha perspectiva para a consulta médica e de enfermagem (...) o que disse foi levado em conta e senti que contribui para a mesma" $(\mathrm{E} 2)$.

O trabalho em equipa, a rápida integração e atitude acolhedora dos profissionais face aos estudantes aumenta-Ihes a sentimento de pertença (o Eu na equipa), facilita as relações interpessoais e esbate as relações de poder, que se estabelecem intra e interequipas.

Em relação à Integração teórico-prática revela-se no discurso oral destes finalistas a percepção que a experiência contribuiu para esbater aquilo que muitas vezes se denomina como o fosso entre a teoria e a prática "a participação no projeto permitiu aferir que aquele conhecimento é pertinente e que pode ser aplicado" (E3). Por outro lado, como há uma componente de inovação e de mudança das práticas que implica a justificação (o porquê?) das atividades em curso, há que identificar o que se sabe sobre o assunto e como o introduzir na clínica (adequação ao contexto) e esta necessidade de utilizar corretamente o conhecimento na ação torna consciente a integração dos conteúdos teóricos nos contextos de clínica, esbatendo a noção que teoria e prática são dois mundos diferentes. Os excertos no discurso destes participante "ver como se pode aplicar na prática o que se aprende na escola" (E6) e "na escola aprendemos, mas nem sempre vemos exemplos práticos tão próximos da teoria" (E8) ilustram que o processo de participação na TS potencia a articulação e integração dos conhecimentos teóricos e práticos. 
Na categoria Tomada de decisão clínica baseada na evidência os futuros enfermeiros destacam como positivos: a negociação da decisão em equipa, o tornar claro qual a intencionalidade por detrás da decisão, a antecipação do resultado, a reflexão sobre a ação e a capacidade de resolução de problemas. A reflexão sobre a ação surge associada à discussão interpares e à segurança. A participação no projeto implicava a reuniões de equipa onde na discussão apareciam questões e dúvidas que implicam a reflexão sobre a intervenção ou sobre o processo para a introduzir na clínica "vendo os aspetos positivos e negativos, decidindo qual a melhor forma de o fazer, prevendo resultados e havia sempre alguém do contra a perguntar os riscos (...) tínhamos que ver todos os ângulos"(E6) e esta reflexão sobre a ação aumentava a segurança. Por outro lado "pensar que poderia ser diferente se fizer assim ou de outra forma" (E3) implicava uma reflexão entre os modos de fazer, o saber dito teórico aprendido nas aulas de predomínio teórico e o que se observava no contexto.

A discussão e a negociação que envolviam as atividades extracurriculares foram transversais à própria consecução dos objetivos do EC "o falarmos abertamente sobre as nossa dúvidas, incertezas fez termos a certeza que os meios implementados eram os certos" (E2), o que aumentou a intencionalidade na execução das intervenções pelos estudantes. $\mathrm{O}$ à vontade para questionar e o sentimento de pertença ao contexto possibilitou a integração de saberes e práticas quotidianas essenciais à tomada de decisão.

No que concerne à categoria - Autoaprendizagem os participantes consideram que a participação Ihes aumentou a responsabilidade sobre os resultados que tinham de obter, conferiu autonomia e motivou-os "saber que há um projeto para o qual o nosso trabalho vai contribuir, mais à frente, motiva para aprender e fazer bem" (E2), "no nosso caso particular a nossa pesquisa [Revisão Integrativa da Literatura] deu resultados que permitiram implementar uma consulta de follow up na unidade [de cuidados intensivos] e futuramente vai levar a uma mudança dos cuidados no serviço (....) há medida que tomamos consciência da importância começamos a trabalhar mais"(E5).

Por último apresentamos a categoria Literacia científica. Nesta os estudantes consideram que aprenderam a transferir conhecimento por forma a permitir a integração de saberes e práticas quotidianas essenciais à construção de uma PBE, "ajudou a pensar no processo de transferir o que aprendemos antes de ir para o estágio para a prestação de cuidados" (E7). Para o desenvolvimento desta competência contribuiu a avaliação e a interpretação dos 
resultados dos estudos, fundamentando as decisões (e o próprio projeto) e fazendo chegar à clínica um conjunto de intervenções clínicas, mais ou menos complexas, que introduziram novos modos de fazer ou produziram mudanças nos atuais modos de fazer. Ainda nesta categoria é de referir que quatro estudantes valorizaram a experiência de participação numas jornadas internacionais com pósteres para a divulgação do projeto.

\section{DISCUSSÃO}

Os autores advogam que os EC têm uma elevada potencialidade enquanto metodologia ativa de ensino-aprendizagem (Heidari \& Norouzadeh, 2015; Baixinho \& Ferreira, 2019). Neste caso quisemos compreender como o envolvimento num outro tipo de atividade extracurricular, mas complementar pode influenciar o desenvolvimento de uma PBE.

Os achados apontam para ganhos no envolvimento no projeto TS, com uma adequada integração teórico-prática potenciada por novas aprendizagens, em contexto, sobre intervenções que solucionam problemas, melhoram cuidados e reduzem o tempo de internamento, promovendo a articulação entre níveis de cuidados (Ferreira et al., 2019). facto de se criar uma experiência eficaz de aprendizagem ajuda na construção do conhecimento de enfermagem e potencialmente contribui para a saúde da comunidade (Jansen et al., 2015; Jong, Meijer, Schout , \& Abma, 2018; Long, Bischoff, \& Aduddell, 2018) e pode potenciar os resultados de aprendizagem do próprio EC.

Esta sinergia entre o EC e a atividade extracurricular é uma mais valia porque como referem Benito et al. (2012) é de extrema relevância que os intervenientes no processo (professores, enfermeiros e estudantes) possam vivenciar o ensino clínico com a visão sobre a aquisição/consolidação de competências e habilidades específicas da Enfermagem, enquanto dão resposta aos resultados de aprendizagem esperados para a licenciatura (Jansen et al., 2015).

Neste estudo, constatamos que a decisão sobre a ação na clínica torna-se mais refletida e discutida na equipa, implicando a leitura, análise e transferência do conhecimento obtido por diferentes métodos e aumentando a segurança na transferência do conhecimento produzido para os cuidados de saúde. Os alunos sentem-se motivados a guiar a sua aprendizagem para dar resposta aos inputs do contexto, direcionando a sua pesquisa em bases de dados para uma resposta adequada e, apesar do acréscimo de trabalho, manifestam entusiasmo e 
motivação porque verificam a curto prazo o seu contributo nas mudanças instituídas nos serviços.

Outras investigações sobre o envolvimento dos estudantes em projetos de PBE e que podem incluir atividades diferentes, desde a participação em investigação, à realização de revisões de literatura com método científico, à colheita e análise de dados, até à divulgação dos resultados com comunicações e participação na redação de artigos científicos, também corroboram que este envolvimento tem ganhos em termos de conhecimento e motivação, até pela constatação que a concretização das atividades que the são solicitadas ajuda no progresso da investigação (Slattery et al., 2016; Jong et al., 2018; Einarsen \& Giske, 2019). Apesar destas potencialidades os resultados de outros estudos constatam que os estudantes apresentam baixos níveis de conhecimento, atitude e intenção de implementar uma PBE (Ashktorab, Pashaeypoor, Rassouli, \& Alavi-Majd, 2015), o que, de certa forma, contrasta com a finalidade da academia na preparação de licenciados competentes para fornecer uma base de qualidade e evidência nos cuidados de enfermagem (Baixinho \& Ferreira, 2019).

Os resultados tendem a confirmar que há aprendizagens e desenvolvimento de competências importantes para uma PBE tais como a tomada de decisão baseada na evidência, o saber questionar, o saber transferir o conhecimento para a clínica, o avaliar investigação, o interpretar resultados e o comunicar ciência. Outros estudos subsidiam estes achados ao recomendarem o envolvimento como uma estratégia importante para a aquisição de conhecimento e desenvolvimento de competências para adesão a uma verdadeira PBE, enquanto futuros profissionais (Jansen et al., 2015; Long, Bischoff, \& Aduddell, 2018).

A análise do discurso aponta para uma intenção em continuar a valorizar a PBE e a introdução dos resultados da investigação na clínica enquanto futuros profissionais. No entanto, não é possível prever esse impacto pela diversidade de contextos clínicos, existentes em Portugal, e com características diferentes desde a produção ao consumo de ciência e porque o intervalo temporal entre a experiência tida e o focus group é curta, o que pode influenciar a intenção. Este aspecto deve ser explorado em estudos futuros.

De referir que, na avaliação do programa de iniciação à investigação desenvolvido por Slattery et al. (2016) os achados apontam para que a exposição precoce, dos estudantes de enfermagem, a diferentes atividades de pesquisa encoraja os recém-formados a incorporar 
a evidência na sua clínica futura e a serem mais proativos na procura de formação pósgraduada. Outros estudos sobre o tema registam as vantagens da participação ativa nas diferentes fases de pesquisa, mas não são claros quanto ao impacto destas experiências após o seu término.

Acima de tudo, a participação condicionou, favoravelmente, o trabalho interdisciplinar e a integração, com os participantes a manifestarem o sentido de pertença e de bem-estar nos serviços. Já Slattery et al. (2016) salientavam que a participação no programa (de PBE) permitiu o conhecimento dos vários papéis e o desenvolvimento de capacidade para trabalhar em equipas interdisciplinares. Aparentemente sem ligação direta à PBE a questão da integração e da comunicação intra e inter equipas é de major importância. Como observam Jansen et al. (2015) os estudantes têm, nestas atividades, a possibilidade de liderar e promover o trabalho em equipa e concomitantemente, desenvolvem competências comunicacionais fundamentais para a prestação de cuidados em contextos de saúde, esperando-se que sejam capazes de as mobilizar no estabelecimento de uma relação terapêutica com o cliente (van Graan, Williams, \& Koen, 2016).

Ressalvamos que este é um estudo exploratório, limitado a um contexto muito especifico, mas cujos achados contribuem para a discussão em torno da ideia de Long, Bischoff e Aduddel (2018) que pesquisar durante a licenciatura é um foco emergente para ajudar os alunos a desenvolver as habilidades e competências necessárias para um profissional de saúde na atualidade.

\section{CONCLUSÕES}

A educação em enfermagem e nas outras disciplinas da saúde deve promover o pensamento crítico, a reflexão e a tomada de decisão segura, garantindo aos utilizadores dos cuidados de saúde o melhor cuidado, com o menor custo. A ideia contida nesta afirmação é uma preocupação internacional e um desafio para uma PBE, em que haja conhecimentos, atitudes e competências dos alunos ao nível da evidência científica.

Este estudo exploratório, inserido num paradigma qualitativo teve como intenção compreender como os estudantes finalistas de enfermagem percepcionam o seu envolvimento numa atividade extracurricular de translação de conhecimento para a clínica, durante o seu estágio de integração à vida profissional. 
A análise do discurso dos participantes neste focus group permitiu a definição de cinco categorias sobre o envolvimento dos estudantes no projeto TS: trabalho interdisciplinar, integração teórico-prática, tomada de decisão clínica baseada na evidência, autoaprendizagem e literacia científica.

Os achados são motivadores para a continuidade do envolvimento dos estudantes no projeto, mas há necessidade de aprofundar, em estudos futuros, a influência da participação neste tipo de atividades no aumento da literacia científica e na utilização da evidência nos primeiros cinco anos de atividade profissional, bem como o contributo deste tipo de metodologia na Educação em Enfermagem para o desenvolvimento das competências necessárias para os enfermeiros de cuidados gerais.

Esta pesquisa tem limitações associadas ao contexto e à própria natureza do estudo que não permite generalizações para outras realidades. O método têm a desvantagem da impossibilidade de saber se a interação em grupo influenciou as respostas individuais. Todavia numa área onde a investigação ainda é escassa a opção por um estudo de natureza qualitativa possibilitou explorar o fenómeno em estudo e as categorias e subcategorias que emergiram possibilitam guiar estudos futuros.

Agradecimentos. Agradecemos às Instituições Parceiras as condições criadas para 0 desenvolvimento deste projeto e em particular aos estudantes envolvidos pelo empenho, dinamismo e capacidade de trabalho.

\section{REFERÊNCIAS}

Ashktorab, T., Pashaeypoor, S., Rassouli, M., \& Alavi-Majd, H. (2015). Nursing Students' Competencies in Evidence-Based Practice and Its Related Factors. Nursing and midwifery studies, 4(4):e23047. doi:10.17795/nmsjournal23047.

Baumbusch, J.L., Kirkham, S.R., Khan, K.B., McDonald, H., Semeniuk, P., Tan, E., \& Anderson, J.M. (2008). Pursuing Common Agendas: A Collaborative Model for Knowledge Translation between Research and Practice in Clínical Settings. Research in Nursing \& Health, 31(2), 130-40. doi: 10.1002/nur.20242.

Baixinho, C.L., Ferreira, Ó., Marques, F.M., Presado, M.H., \& Cardoso, M. (2017). Transição segura: um projeto da transferência do conhecimento para a prática clínica. In Costa AP, Sánches-Gómez MC, Cilleros M.V.M. A prática na Investigação Qualitativa: exemplos de estudos (pp.57-80). Oliveira de Azeméis: Ludomédia.

Baixinho, C.L., \& Ferreira, Ó. (2019). Aprender em projetos de translação de conhecimento durante o ensino clínico. In: A.P. Costa, S.O. Sá, P: Castro, D.N., Souza, Atas do $8^{\circ}$ Congresso lbero-Americano em Investigação Qualitativa. Oliveira de Azeméis: Ludomedia, pp.159-68.

Benito, G.A.V., Tristão, K.M., Paula, A.C., Santos, M.A., Ataide, L.J., \& Lima, R.C.D. (2012). Desenvolvimento de competências gerais durante 0 estágio supervisionado. Rev Bras Enferm, 65(1),172-8. doi: https://doi.org/10.1590/S0034-71672012000100025 
Bloor, M., Frankland, J., Thomas, M. \& Robson, K. (2001). Focus groups in social research. London: Sage.

Einarsen, K.A., \& Giske, T. (2019). Nursing students' longitudinal learning outcomes after participation in a research project in a hospital. IPDJ. 9(1), 4. doi: https://doi.org/10.19043/ipdj.91.004.

Ferreira, E.M., Lourenço, O.M., Costa, P.V., et al. (2019). Active Life: a project for a safe hospital-community transition after arthroplasty. Revista Brasileira de Enfermagem, 72(1), 147-153. doi: https://dx.doi.org/10.1590/0034-7167-2018-0615.

Jansen, D.A., Jadack RA, Ayoola AB, Doornbos MM, Dunn SL, Moch SD, Moore EM, \& Wegner GD. (2015). Embedding Research in Undergraduate Learning Opportunities. West J Nurs Res., 37(10),1340-58. doi: $10.1177 / 0193945915571136$.

Jong, G., Meijer, E., Schout, G., \& Abma, T. (2018). Involving Undergraduate Nursing Students in Participatory Health Research: Implications from the Netherlands. $J$ Prof Nurs; 34(6), 507-513. doi: https://doi.org/10.1016/j.profnurs.2018.04.001.

Heidari, M. R., \& Norouzadeh, R. (2015). Nursing students' perspectives on clinical education. Journal of Advances in Medical Education \& Professionalism, 3(1), 39-43.

Kitson, A., Brook, A., Harvey, G., Jordan, Z., Marshall, R., O’Shea, R., \& Wilson, D. (2018). Using complexity and network concepts to inform healthcare knowledge translation. International Journal of Health Policy and Management, 7(3), 231-243.

Krueger, R.A. \& Casey, M.A. (2014). Focus Groups: A Practical Guide for Applied Research. (5 th $^{\text {ed }) . ~ N e w ~}$ Delhi: Sage.

Long, A., Bischoff, W.R., \& Aduddell, K. (2018). Research Prescription for Undergraduate Students: Research Mentoring in a Small Liberal Arts University. $J$ Prof Nurs, 35(3), 170-3. doi: https://doi.org/10.1016/i.profnurs.2018.12.007.

Mena-Tudela, D., González-Chordá, V.M., Cervera-Gasch, A., Maciá-Soler, M.L., \& Orts-Cortés, M. I. (2018). Effectiveness of an Evidence-Based Practice educational intervention with second-year nursing students. Rev Latino-Am Enfermagem, 26, e3026. doi:https://dx.doi.org/10.1590/1518-8345.2502.3026.

Paniagua, D.V., Ribeiro, M.P.H., Correia, A.M., et al. (2018). Project K: Training for hospital-community safe transition. Rev Bras Enferm, 71(Suppl. 5), 2264-71. doi:https://dx.doi.org/10.1590/0034-7167-2018-0190.

Silva, I.S., Veloso, A.L., \& Keating, J.B. (2014). Focus group: Considerações teóricas e metodológicas. RLE, 26(26), 175-90. Available from: https://revistas.ulusofona.pt/index.php/rleducacao/article/view/4703.

Slattery, M. J., Logan, B. L., Mudge, B., Secore, K., von Reyn, L. J., \& Maue, R. A. (2016). An Undergraduate Research Fellowship Program to Prepare Nursing Students for Future Workforce Roles. Journal of professional nursing : official journal of the American Association of Colleges of Nursing, 32(6), 412-420. doi: https://doi.org/10.1016/j.profnurs.2016.03.008.

Sun, J.-H., Liu, J.-E., Wu, Y., \& Li, S.J. (2014). The Effects Of The Student-Centered Clinical Nursing Practice Mode Based On The Action Research For Clinical Practicum Of Undergraduate Students In Beijing, China. Procedia Soc Behav Sci; 141, 839 - 45. doi: 10.1016/j.sbspro.2014.05.146.

Van Graan, A.C., Williams, M. J.S., \& Koen, M. P. (2016). Professional nurses' understanding of clinical judgement: A contextual inquiry. Health SA Gesondheid, 21, 280-293. doi: https://doi.org/10.1016/j.hsag.2016.04.001. 